\title{
Analysis on Patent Application in Piezoelectric Inkjet Printing Head Based on Derwent Innovations Index
}

\author{
Wang $\mathrm{Yi}^{1, \mathrm{a}^{*}}$, Mao $\mathrm{Di}^{2, \mathrm{~b}}$ and Dou Ruiling ${ }^{3, \mathrm{c}}$ \\ ${ }^{1}$ Suzhou Institute of Nano-tech and Nano-bionics, Chinese Academy of Sciences, Suzhou, China \\ ${ }^{2}$ Wuhan Branch of the National Science Library, Chinese Academy of Sciences, Wuhan, China \\ ${ }^{3}$ Suzhou Institute of Nano-tech and Nano-bionics, Chinese Academy of Sciences, Suzhou, China \\ aywang2008@sinano.ac.cn, ${ }^{b}$ maod@whlib.ac.cn, ${ }^{c}$ rldou2006@sinano.ac.cn
}

Keywords: Piezoelectric inkjet printing head; 3D print; patent analysis; Derwent Innovations Index

\begin{abstract}
This paper analyzed the overall patent situation of global piezoelectric inkjet printing head technology, the layout of relevant technological field and the main competitive regions and applicants from the developed background of the global piezoelectric inkjet printing head technology. Based on the analysis of overall situation analysis, we has especially analyzed the patents of the regions with the best advanced technology and high market demand in Japan; than disclosed the intellectual property risk faced by piezoelectric inkjet printing head technology by the comparative analysis and function analysis of various research units.
\end{abstract}

\section{Introduction}

There are two kinds of inkjet printing heads which are divided by different operating principles: the first one is the continuous inkjet printing head, which can sprays ink droplets continuously. Some of ink sprayed on the media selectively and the rest are back to the system for recycling. Another one is the pulsing inkjet printing head (jetting the ink according to the requirements), from which the ink droplets sprayed by the discontinuous electrical signals (waveform). In this article, we focus on the piezoelectric inkjet print head which is based on the DOD technologies. There are many advantages of this kind print head, for example, it has long service lifetime, and it can be applied to different types of ink, including UV inkjet ink owning high viscosity and ceramic glaze, etc.; but the cost of manufacturing is very high. At present, there are many companies to make piezoelectric ink jet head, such as Epson, Cernet, Kyocera, Toshiba, konica minolta, panasonic, Trident, and so on. Piezoelectric ink jet head has many patent technology, including Face Shooters, Piston Structures and Moving Wall Structures, etc.

As the core component in inkjet printing device, printing head has gained a great development in recent years. Due to the piezoelectric inkjet head can be applied to various kinds of ink, including high viscosity UV inkjet ink and ceramic glaze, etc., and to the $3 \mathrm{~d}$ printing, this article studied the technology development of the piezoelectric inkjet printing head from the perspective of patent analysis, in order to provide support and help for future research.

\section{Data Source and Research Method}

(1) The analytical data comes from Derwent Innovations Index, DII. (2) Data retrieval time: researching DII data base and getting 4799 public patents from 1989 to 2014 on February 12, 2013 (3) Retrieval strategy: retrieval type is piezo* inkjet head*. (4) Adopted analytical tools : TDA, Excel, and so on.

\section{Technology Lifecycle of Piezoelectric Inkjet Printing Head Technology Patents}

Technology lifecycle is made up of stages in germination, growth period, maturation period and phase of decline. Based on the number of patent application about piezoelectric inkjet printing head 
technology and the number of patent applicant each year, Figure 1 shows the development process of patents related to piezoelectric inkjet printing head technology. It reveals that patent related to piezoelectric inkjet printing head technology is in its infancy before 1995, then, it enters the stage of technological rapid growth between 1995-2005. From 2005 untill now, it has been becoming mature phase.

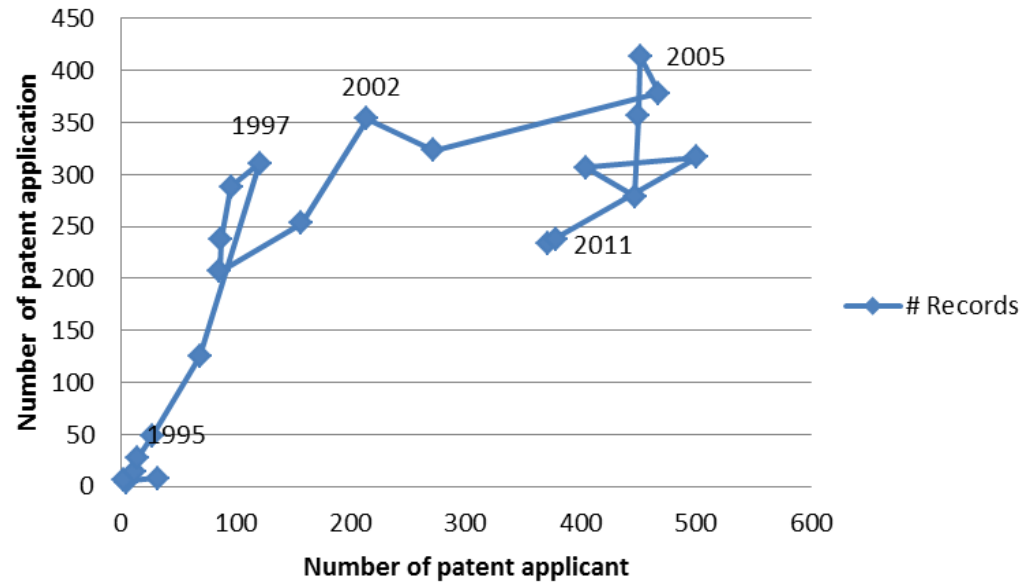

Figure 1: Technology Lifecycle of piezoelectric inkjet printing head technology patents

\section{Distribution of IPC on Piezoelectric Inkjet Printing Head Technology}

Through analysis on the patent of piezoelectric inkjet printing head based on IPC, we can understand and analyze the technological fields and technological key points related to piezoelectric inkjet printing head. Figure 2 lists the top 15 patent classifications, and it can visually present the first and second patent application quantities, which are the hotspots of the technology.

The meaning of B41J-002 is typewriters or selective printing mechanisms characterized by the printing or marking process for which they are designed. And H01L-041 is piezoelectric devices in general; Electrostrictive devices in general; Magnetostrictive devices in general; Processes or apparatus specially adapted for the manufacture or treatment thereof or of parts thereof.

The patent technology of piezoelectric inkjet printing head focuses on the following aspects: (1) structures, processes and components of the printer, the main classification codes of which are B41J-002 and B41J-029; (2) piezoelectric devices, electromotor processes and their manufacturing methods or equipments: H01L-041, H02N-002, H01L-021, C23C-014 and C04B-035 are the main classification codes; (3) duplicating or liquid spouting methods, the main classification codes are B05C-005 and B41M-005; (4) ink materials, such as printing ink, ceramics, and so on, which have two main classification codes of C09D-011 and C04B-035.

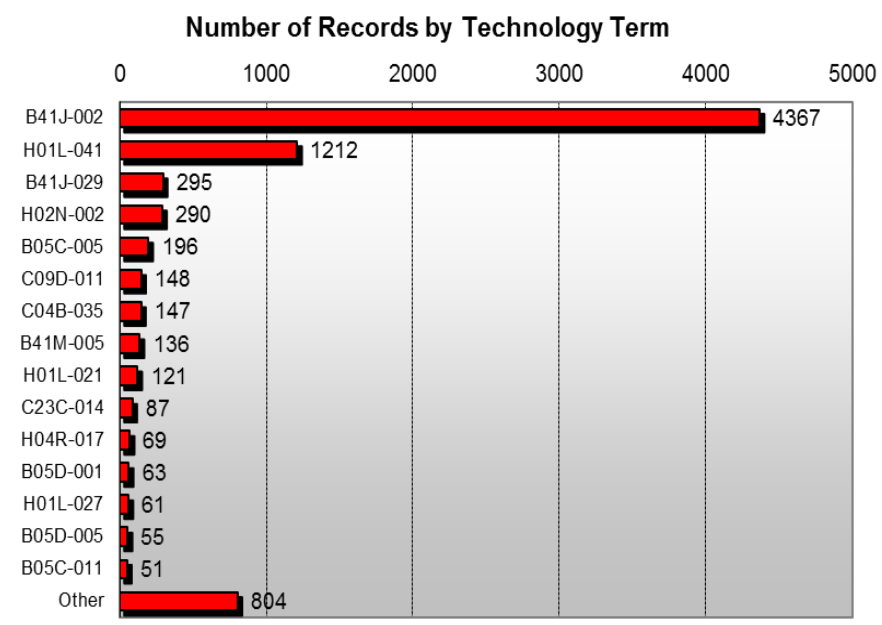

Figure 2 Distribution of IPC classifications of piezoelectric inkjet printing head. 


\begin{tabular}{cccc}
\hline IPC & NO. & Related years & $\begin{array}{c}\text { Quantity Percent of recent 3 } \\
\text { years }\end{array}$ \\
\hline B41J-002 & 4367 & $1989-2012$ & $11 \%$ of 4367 \\
H01L-041 & 1212 & $1989-2012$ & $12 \%$ of 1212 \\
B41J-029 & 295 & $1994-2011$ & $16 \%$ of 295 \\
H02N-002 & 290 & $1989-2012$ & $5 \%$ of 290 \\
B05C-005 & 196 & $2000-2012$ & $23 \%$ of 196 \\
C09D-011 & 148 & $1998-2011$ & $9 \%$ of 148 \\
C04B-035 & 147 & $1995-2011$ & $14 \%$ of 147 \\
B41M-005 & 136 & $1989-2011$ & $8 \%$ of 136 \\
H01L-021 & 121 & $1995-2012$ & $23 \%$ of 121 \\
C23C-014 & 87 & $1999-2012$ & $17 \%$ of 87 \\
\hline
\end{tabular}

Table 1 Situation of IPC about the number of top 10 patent application.

\section{Countries/Regions Distribution of Patents of Piezoelectric Inkjet Printing Head}

\subsection{Countries / regions distribution of patents}

Figure 3 provides top 10 countries / regions which have more patent application quantities of piezoelectric inkjet printing head. From that figure, we can see that the nations/regions that have more relevant patent application quantities of piezoelectric inkjet printing head concentrate on Japan (JP), United States (US), South Korea (KR). Especially in Japan, the patent acceptance quantities and the priority paten quantities occupy about $90 \%$ of the total patents in the world, which is far ahead of other nations / regions, and shows Japan's leader status of piezoelectric inkjet printing head technology in the world.

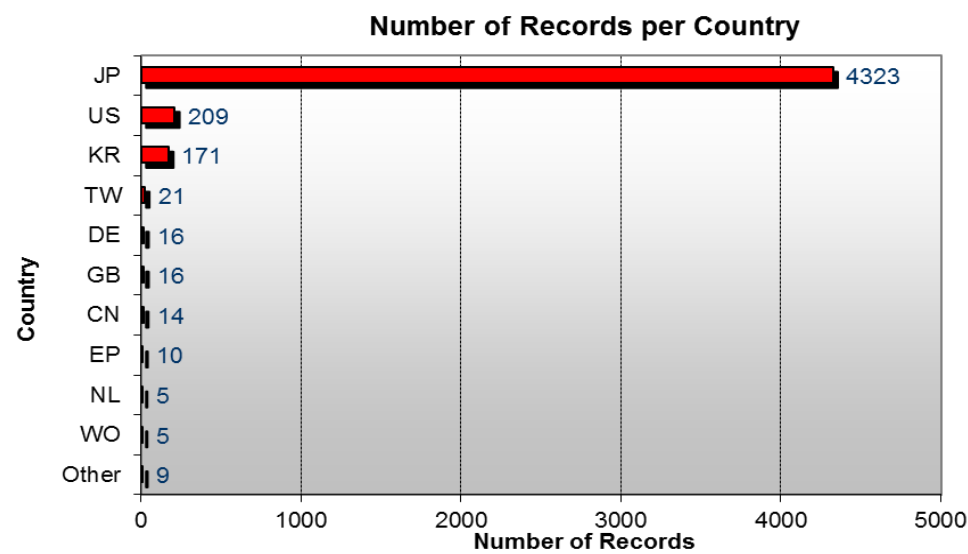

Figure 3: Top 10 countries / nation of acceptance quantity of patent of piezoelectric inkjet printing head. 


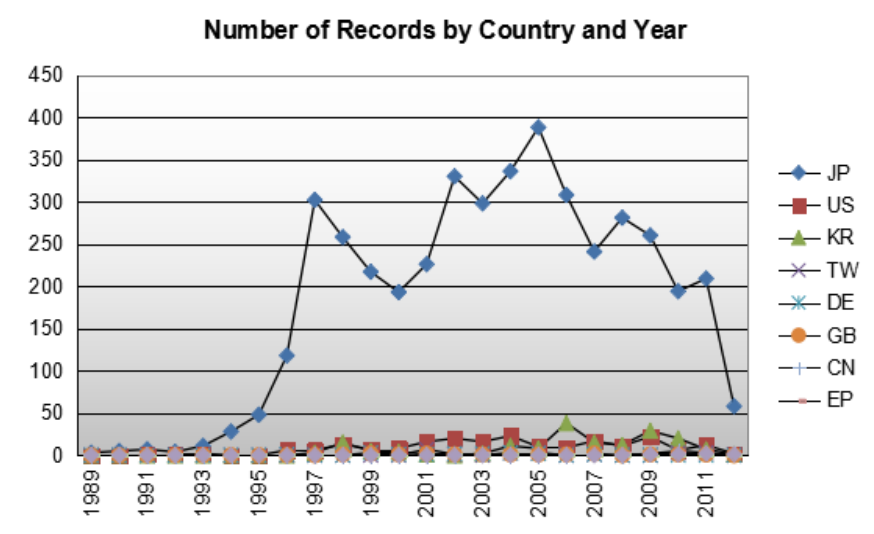

Figure 4: Annual patent quantity distribution situa-tion of top 10 countries/ regions.

\subsection{IPC distribution between different countries / regions}

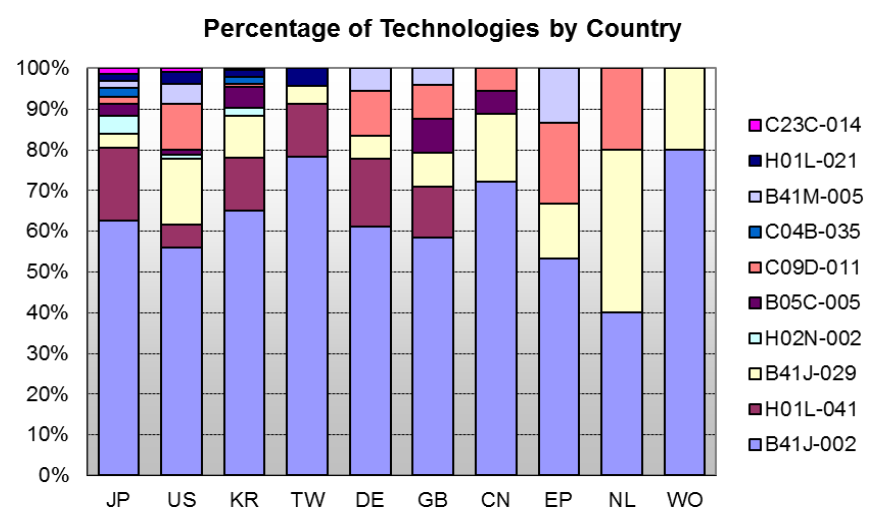

Figure 5 Technological layout of main countires / regions in the field of piezoelectric inkjet printing head

Figure 5 and Table 2 shows the technological layout of top 10 countries / regions, which is Japan, United Sates, South Korea, China Taiwan, Germany, Great Britain, China, European patent bureau, Netherlands and International patent, they are the leaders in the field of piezoelectric inkjet printing head. The analysis is based on the priority patent application data. It can be seen that the technology structure of main countries/ regions is similar to each other., and patents are mostly relative to B41J-002, H01L-041, B41J-029, and C09D-011 in piezoelectric inkjet printing head, which are the mechanical properties, design performance, piezoelectric device features, fabricated equipment, printing ink, and so on. Specifically speaking, the technological distributions of top 3 most important countries / regions are:

(1) Japan(4323) mainly focuses on those fields as B41J-002, H01L-041, B41J-029, B05C-005, C09D-011 and so on.Besides, the patents of H02N-002 (piezoelectric effect motor), C04B-035 (ceramic composition), and C23C-014 (material coating) mainly concentrate on Japan and it is rare in other nations.

(2) United States(209) mainly focus on such fields as B41J-002, B41J-029, C09D-011, and so on, which are the mechanical features, design performance, parts and accessories of printers, and printing ink.

(3) The patents of South Korea (171) are divided into such fields as B41J-002, H01L-041, B41J-029, and so on, which are the mechanical features, parts and accessories of printers, and piezoelectric devices. besides, it also pays attention to B05C-005.

\subsection{Comparison of patent family in main countries/ regions}

Based on the statistics of the patent family of main countries/ regions, from Table 3 it shows that the patent family size of England and Germany are more larger the other countries, and all the other countries have no difference. 


\begin{tabular}{lcccccccc}
\hline Nation & JP & US & KR & TW & DE & GB & CN & EP \\
\hline No.Patent family & 9051 & 808 & 566 & 46 & 78 & 139 & 41 & 38 \\
No.Patent & 4323 & 209 & 171 & 21 & 16 & 16 & 14 & 10 \\
Patent family size & 2.09 & 3.87 & 3.31 & 2.19 & 4.88 & 8.69 & 2.93 & 3.8 \\
\hline
\end{tabular}

Table 3. Comparison of patent family in main countires/ regions

\section{Analysis of Patent Applicant of Piezoelectric Inkjet Printing Head}

\subsection{Important patent applicant}

Among the top 10 organization applicant in Figure 6, organizations from Japan have leading advantage absolutely. The tenth comes from South Korea, and the other organizations are all from Japan; the patent applicant in top 10 Japanese organizations takes up $78.0 \%$ of all the patent application quantity and $83.8 \%$ of patent application quantity of main organizations (top 25 ),respectively. The top 10 patent applicants are: SEIKO EPSON Corporation, Fuji Film Holdings, Brother Kogyo, Japan Ricoh Corporation, KYOCERA Corporation, Konica Corporation, Panasonic Corporation, Hitachi Corporation, Canon Corporation, and Samsung Electronics.

\begin{tabular}{llllllllll}
\hline IPC & JP & US & KR & TW & DE & GB & CN & EP & WO \\
\hline B41J-002 & 4004 & 134 & 155 & 18 & 11 & 14 & 13 & 8 & 4 \\
H01L-041 & 1158 & 13 & 31 & 3 & 3 & 3 & 0 & 0 & 0 \\
B41J-029 & 219 & 39 & 24 & 1 & 1 & 2 & 3 & 2 & 1 \\
H02N-002 & 283 & 2 & 5 & 0 & 0 & 0 & 0 & 0 & 0 \\
B05C-005 & 178 & 3 & 12 & 0 & 0 & 2 & 1 & 0 & 0 \\
C09D-011 & 110 & 27 & 2 & 0 & 2 & 2 & 1 & 3 & 0 \\
C04B-035 & 143 & 0 & 4 & 0 & 0 & 0 & 0 & 0 & 0 \\
B41M-005 & 120 & 12 & 0 & 0 & 1 & 1 & 0 & 2 & 0 \\
H01L-021 & 109 & 7 & 4 & 1 & 0 & 0 & 0 & 0 & 0 \\
C23C-014 & 84 & 2 & 1 & 0 & 0 & 0 & 0 & 0 & 0 \\
\hline
\end{tabular}

Table 2. Technological layout of main countires / regions in the field of piezoelectric inkjet printing head

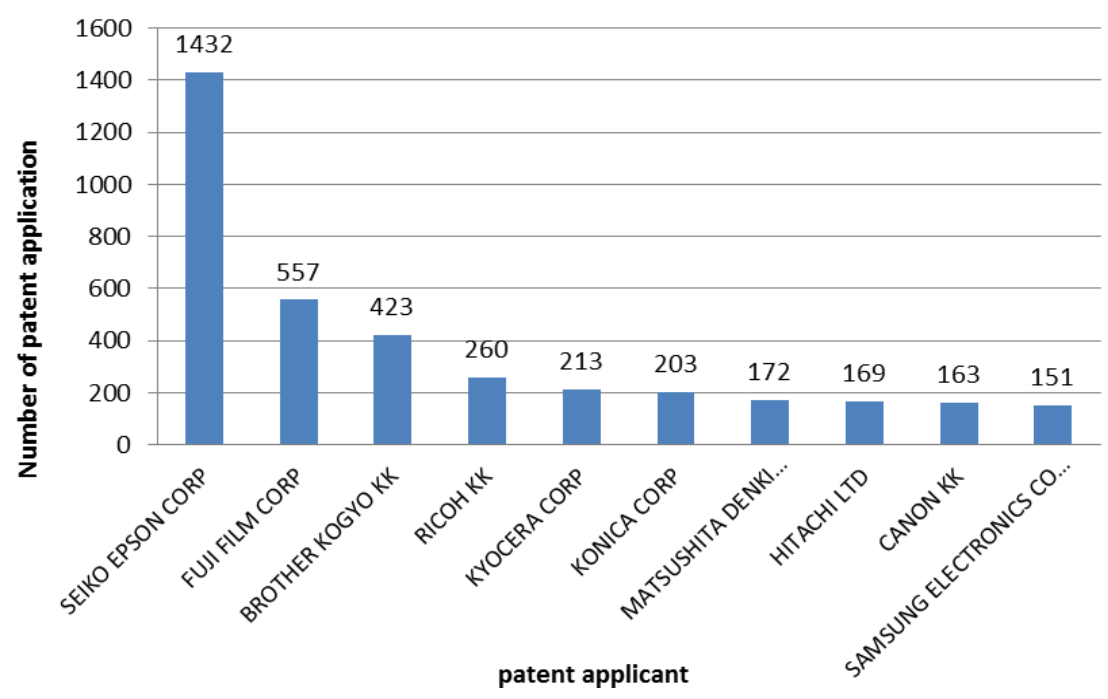

Figure 6 Top 10 organization applicants

\subsection{Patent protection strategies of main patent applicants}

From Table 3, we can see that the patent application area of main patentees concentrates on their own nations. Among other nations, the patent application with the highest proportion is Fuji Corporation, with 557 patents, including 23 patents of United States, 6 patents of South Korea, 1 patent of Germany and European patent bureau, respectively; Epson Corporation has 5 patents in China, 5 in United States, 1 in Germany and European patent bureau, respectively. Brother Corporation has 8 patents in United States, 1 in Germany. 


\begin{tabular}{|c|c|c|c|c|c|c|c|}
\hline Patentee & No.Patent & JP & US & KR & $\mathbf{C N}$ & DE & EP \\
\hline Seiko Epson & 1432 & 1431 & 5 & 1 & 5 & 1 & 1 \\
\hline FijiFilm Corporation & 557 & 539 & 23 & 6 & & 1 & 1 \\
\hline Brother Kogyo & 423 & 422 & 8 & & & 1 & \\
\hline Ricoh Corporation & 260 & 260 & & & & & \\
\hline KYOCERA Corporation & 213 & 212 & 1 & & & & \\
\hline Konica & 203 & 203 & & & & & \\
\hline Panasonic & 172 & 172 & & & & & \\
\hline Hitachi & 169 & 168 & 7 & & & & \\
\hline Canon & 163 & 163 & 3 & 1 & & & \\
\hline Samsung & 151 & & 1 & 151 & & & \\
\hline
\end{tabular}

Table 3: Patent protection strategy comparison of main patentees of piezoelectric inkjet printing head.

\section{Conclusion}

Piezoelectric inkjet printing head is the core component of the inkjet printing device, art of inkjet printing tends towards more preciser on ink dropping control and higher frequency on ink dropping.

The whole technical route goes through the infancy stage(1989-1995),growth phase(1995-2005) and maturation period(2005-2012). IPC classifications center on B41J-002 and H01L-041, and the patentees are interesting on mechanical properties, design performance, piezoelectric device features, fabricated equipment, printing ink, and so on. Japan is the earliest country which has been setting about to research piezoelectric inkjet printing head from 1989, and the patent applicant which in it has more superiority and competitive than the other ones. The patentees of Top 9 are all coming from Japan. But we can see that the companies in Japan hasn't apply the patent widely in the other area via PCT, they are only pay attention to the native market.

Through analysis of the patent data based on Derwent Innovations Index, we study the intelligent maps of piezoelectric inkjet printing head technology all around the world, to providing powerful support to the choice of future research orientation, analysis of intellectual property rights and industrial development.

\section{References}

[1] Qiao Yongzhong. 2011. The factors affecting the patent maintenance time. Scientific research management,32(7):143-149.

[2] Wan Zheng, 2011. On evaluation of patent asset. Anhui Technology. (5):35-37.

[3] Liu Yubo, 2011. Analyze and define corporation patent strategies based on technological innovation mode". Chinese inventions and patent. (7):34-35. 